\title{
Treatment of tracheoesophageal fistula after laryngectomy by a customized tracheal prosthesis
}

\section{Running title}

Tracheoesophageal fistula treatment

\author{
Authors \\ Simon Andreas Mueller, MD ${ }^{1}$, Sylvia Dehnbostel ${ }^{2}$, Falk Dehnbostel, Roland Giger, MD ${ }^{1}$ \\ ${ }^{1}$ Department of Oto-Rhino-Laryngology, Head and Neck Surgery, Inselspital, Bern University Hospital, \\ University of Bern, 3010 Bern, Switzerland \\ ${ }^{2}$ Epithetik-Swiss - Institute for Epithetics, 8873 Amden, Switzerland
}

\section{Funding and conflicts of interest}

No external funding was received. The authors have no conflicts of interest to disclose.

\section{Corresponding author}

Simon A. Mueller, MD

Department of Oto-Rhino-Laryngology, Head and Neck Surgery

Inselspital, Bern University Hospital, Freiburgstrasse 10, 3010 Bern, Switzerland.

Email: simon.mueller@insel.ch, Phone: +41 31632 0418, Fax: +41 316328809 


\section{KEY WORDS}

Tracheoesophageal fistula treatment, laryngectomy, silicone prosthesis, voice prosthesis 


\section{INTRODUCTION}

The management of persistent tracheoesophageal fistulas (TEF) after laryngectomy in previously irradiated patients is challenging. A persistent TEF can develop spontaneously or result from the enlargement of a tracheoesophageal puncture (TEP) harboring the voice prosthesis (VP) (1). Patients treated with radiotherapy are especially at risk of TEP enlargement, with an incidence as high as $17 \%$ after pre-laryngectomy and $27 \%$ after postlaryngectomy radiotherapy (2). A TEF or enlarged TEP lead to leakage of fluids and food into the trachea. In case of an enlarged TEP, spontaneous shrinking or closing may be achieved by removing the VP. This requires the insertion of a tracheal cannula with inflated cuff and feeding via nasogastric or percutaneous gastric tube to avoid aspiration. Other conservative measures include placement of an anterior collar around the VP, and the injection of fat or collagen to corset the TEP (1). When conservative treatments fail, closure of an enlarged TEP can be achieved by purse string suture or more complex closing techniques, using pedicled or free flap reconstructions $(3,4)$.

When surgery is not successful or impossible because of comorbidities, options are limited. Herzog et al. have described TEF closure by means of customized pharyngoesophageal stents $(5,6)$. These stents are usually inserted in general anesthesia and cannot be removed and cleaned by the patient. They may also hinder physiological peristalsis and carry the risk of food impaction.

We describe the development of a customized removable tracheal prosthesis intended to close the TEF, allowing oral nutrition without aspiration and phonation with an incorporated VP. 


\section{MATERIALS AND METHODS}

\section{Case report}

A 52-year old man with a history of cigarette smoking, substance use, alcohol dependence, and important co-morbidities such as chronic obstructive pulmonary disease, hepatitis C, HIV-infection and general cachexia, was treated with radiotherapy for a right vocal fold cancer in 2011. Due to recurrence, he underwent salvage total laryngectomy and bilateral neck dissection in 2012. To decrease the risk of TEF formation, a pectoralis major myofascial flap was placed on the pharyngeal suture to interpose non-irradiated tissue between the neopharynx and the skin (7). The TEP and VP insertion were performed during the same operation. Six months later, gradual atrophy of the tissue around the VP occurred with consecutive periprosthetic leakage of saliva. The VP was removed and the patient was nourished through a percutaneous gastric tube. To avoid pulmonary aspiration, the patient had to wear a cuffed tracheal cannula. However, conservative measures did not lead to narrowing or closure of the TEF and it eventually reached a size of $2.0 \times 1.5 \mathrm{~cm}$ (Figure 1). Surgical closure of the TEF by radial forearm flap was proposed, but given the co-morbidities and considerable perioperative risks, the patient objected to surgery, although his quality of life was severely affected. We therefore developed the idea of a customized tracheal prosthesis to close the TEF, permitting oral nutrition and speech rehabilitation.

\section{Casting of the tracheostomy and modeling of the prosthesis}

A schematic drawing of the prosthesis is shown in Figure 2. The first step is the elaboration of a silicone cast of the tracheostomy, the proximal trachea and the TEF in general anesthesia (Figure 3). The patient is ventilated over a cuffed tracheal cannula preventing aspiration of liquid silicone. We use a Sengstaken tube (Rüsch, Teleflex 
Incorporated, Wayne, PA, USA) to seal the esophagus during the procedure, with its long proximal cuff lying behind the TEF. A layer of Vaseline is applied on the part of the cuff of the Sengstaken tube that is visible through the TEF to prevent sticking of the liquid silicone. The tracheostomy and proximal trachea are filled with two-part silicone (StecoForm ${ }^{\circledR}$ Flex, steco-system-technik, Hamburg, Germany), which is allowed to harden for 5 minutes before it is removed with the still inflated tracheal cannula (Figure 4A and 4B). After removal of the Sengstaken tube, the cast is inserted again and its anatomic accuracy verified by flexible esophagoscopy.

Then, a wax model of the prosthesis matching the silicone cast is elaborated, with sockets for the VP, the moisturizing filter and bails for the fixation band. A cast out of plaster is formed using the wax model. The prosthesis is molded in the plaster cast using FX-502 Silicone-Elastomer® (Factor II, Lakeside, AZ, USA) around a tubular placeholder which creates the lumen of the prosthesis (Figure 4C). Polymerization of the silicone is achieved by heating the prosthesis to 100 degrees Celsius in a hot air sterilizer for 90 minutes. Finally, the socket for the VP is perforated and a Provox ${ }^{\circledR}$ Vega $^{\mathrm{TM}}$ prosthesis (Atos Medical, Sweden) inserted. If necessary, minor changes to the shape of the prosthesis can be made after an initial fitting.

\section{RESULTS}

Figure 5 shows the finished prosthesis. The prosthesis is very well tolerated by the patient who wears it day and night since March 2013. The sealing of the TEF is sufficient to enable oral nutrition. The leak tightness can be verified by flexible tracheoscopy through the prosthesis lumen while the patient is swallowing a colored liquid and solid food. No aspiration pneumonia has occurred since the prosthesis was implemented. 
The patient removes and reinserts the prosthesis by himself for cleaning with ordinary soap water once a day. The phonation with the VP is comparable to that of other laryngectomees. Tightness of the prosthesis around the VP is enduring and periprosthetic leakage never occurred. The possibility to remove the prosthesis facilitates cleaning of the VP. The initial VP remained functional until the whole prosthesis had to be replaced after two years due to important fungal colonization. A new prosthesis was molded using the initial plaster cast. Three years after the first fitting, leaking occurred due to a slight enlargement of the TEF and a second model was elaborated in identical technique.

\section{DISCUSSION}

The presented technique is an innovative approach to close TEF by means of a customized silicone tracheal prosthesis.

Salvage laryngectomy after radiotherapy bears a high risk for the development of TEF (2). When conservative measures fail and surgical closure is not feasible, the affected patients permanently rely on enteral feeding and the use of a VP is impossible. Their quality of life is therefore severely impaired.

Our removable customized tracheal prosthesis is intended to seal the TEF over a long period of time. Oral uptake of nutrition and prosthetic speech is possible, which improves patients' daily life considerably. Besides a fungal colonization of the prosthesis, no other side effects like proliferation of granulation tissue, dislocation, leakage and discomfort were observed for 3 years.

Other authors have described sealing of TEF with customized pharyngoesophageal stents. Herzog et al. use a different type of prosthesis featuring an additional pharyngoesophageal lining in the shape of a tube or a plate, anchoring the prosthesis in the 
TEF $(5,6)$. An additional esophageal tube may simultaneously serve as a stent to bridge a stenosis. Their technique allowed oral feeding and prosthetic speech in some cases.

There are several advantages to removable prosthesis limited to the trachea. First, insertion is easy and does not require general anesthesia. In case of dislocation, the prosthesis can easily be put back into position. Second, maintenance of both, the prosthesis and the VP is easy, as it can be removed. Furthermore, minor adjustments to the shape of the prosthesis can be made after an initial fitting, for example if the patient feels discomfort because of too much pressure on the tissue. Third, since the prosthesis presented here is confined to the trachea, it does not affect the physiological properties of the esophagus. Stenting of the esophagus is much more likely to impair peristalsis and stents are prone to food impaction. Self-expansible esophageal stents have also been used to seal TEF, but they have similar adverse effects on the esophageal physiology. Additionally, they carry the risks of further pressure-induced enlargement of the TEF, perforation, stent migration, hemorrhage and pain (8). Our removable prosthesis proves that stenting of the esophagus from inside is not necessary to achieve sufficient sealing.

Possible complications of any prosthesis for closure of a TEF are fungal colonization and further enlargement of the TEF caused by friction on the surrounding tissue, as it was the case in our patient after three years. Nevertheless, the benefits of the prosthesis and the positive effect on the quality of life clearly outweigh these problems.

In conclusion, this novel customized tracheal prosthesis offers a safe and valuable alternative for the treatment of selected cases of TEF. The customized shape of the prosthesis allows optimal sealing and efficiently prevents aspiration. For patients, maintenance of the prosthesis is easy and they benefit from the possibility to eat and speak using the incorporated VP. 


\section{REFERENCES}

1. Hutcheson KA, Lewin JS, Sturgis EM, Kapadia A, Risser J. Enlarged tracheoesophageal puncture after total laryngectomy: A systematic review and metaanalysis. Head Neck. 2011;33(1):20-30.

2. Hutcheson KA, Lewin JS, Sturgis EM, Risser J. Multivariable analysis of risk factors for enlargement of the tracheoesophageal puncture after total laryngectomy. Head Neck. 2012;34(4):557-67.

3. Jacobs K, Delaere PR, Poorten VLM Vander. Submucosal purse-string suture as a treatment of leakage around the indwelling voice prosthesis. Head Neck. 2008;30(4):485-91.

4. Mobashir MK, Basha WM, Mohamed AES, Anany AM. Management of persistent tracheoesophageal puncture. Eur Arch Otorhinolaryngol. 2014;271(2):379-83.

5. Herzog M, Greiner I. Treatment of large pharyngotracheal fistulas after laryngectomy by a novel customized pharyngeal stent. Eur Arch Otorhinolaryngol. 2011;268(5):74754.

6. Herzog M, Lorenz KJ, Glien A, Greiner I, Plontke S, Plößl S. Treatment of tracheopharyngeal and tracheo-oesophageal fistulas following laryngectomy and fistula classification based on individual silicone casts. Eur Arch Otorhinolaryngol. 2015;272(10):2961-8.

7. Anschütz L, Nisa L, Elicin O, Bojaxhiu B, Caversaccio M, Giger R. Pectoralis major myofascial interposition flap prevents postoperative pharyngocutaneous fistula in salvage total laryngectomy. Eur Arch Otorhinolaryngol. 2016;273(11):3943-9.

8. Battaglia G, Antonello A, Realdon S, Cavallin F, Giacomini F, Ishaq S. Feasibility, efficacy and safety of stent insertion as a palliative treatment for malignant strictures in 
the cervical segment of the esophagus and the hypopharynx. Surg Endosc. 2016;30(1):159-67.

\section{FIGURE LEGENDS}

Figure 1: $\quad$ Tracheostomy and tracheoesophageal fistula (TEF).

Figure 2: Schematic illustration of the removable tracheal prosthesis. 1, neopharynx; 2, prosthesis; 3, tracheoesophageal fistula; 4, esophagus; 5, trachea

Figure 3: Schematic illustration of the elaboration of the silicone cast of the tracheostomy and the tracheoesophageal fistula. 1, silicone cast; 2 , tracheal cannula with inflated cuff; 3, liquid silicone; 4, Sengstaken tube; 5, tracheoesophageal fistula

Figure 4: Casting of the tracheostomy. (A) Filling of the tracheostomy with liquid silicone. (B) Hardened silicone cast of the tracheostomy and the tracheoesophageal fistula (TEF) with tracheal canula. (C) Molding of the prosthesis in casts made out of plaster. The plaster was formed from a wax model of the prosthesis.

Figure 5: $\quad$ Finished prosthesis. (A) Prosthesis with voice prosthesis and moisturizing filter. (B) Inserted prosthesis with moisturizing filter and bails for the fixation strap. 